Revistade
Economild
Contemporâned

\title{
OPORTUNIDADES TECNOLÓGICAS E DIVERSIFICAÇÃO DAS FIRMAS EM TECNOLOGIAS DE MITIGAÇÃO DAS MUDANÇAS CLIMÁTICAS
}

\author{
Paulo Henrique Assis Feitosa $a^{a}$ \\ Renato de Castro Garcia ${ }^{b}$
}

a Professor da Escola de Comunicações e Artes da Universidade da São Paulo (USP). São Paulo, SP, Brasil. ORCID: https://orcid.org/0000-0002-2388-7543.

${ }^{\text {b }}$ Professor do Instituto de Economia da Universidade Estadual de Campinas (Unicamp). Campinas, SP, Brasil. ORCID: https://orcid.org/0000-0001-9739-1658.

\author{
Recebido em 14 maio 2017 \\ Aceito em 04 junho 2021 \\ Received on 14 May 2017 \\ Accepted on 4 June 2021
}

RESUMO: Este artigo tem como objetivo investigar se a diversificação em tecnologias de mitigação das mudanças climáticas é afetada por alterações nos níveis de oportunidades tecnológicas da firma. A partir da constituição de um banco de dados original com de patentes relacionadas a tecnologias de mitigação das mudanças climáticas, foram examinados 120 grupos de tecnologias de todo o mundo e o portfólio de patentes registradas entre os anos 1990-2009 por 436 companhias. Os resultados sugerem uma relação não linear em formato de "U" invertido entre o nível de oportunidade tecnológica e o grau de diversificação das firmas. Assim, este artigo argumenta que a redução da diversificação a partir de determinado ponto da curva pode ser interpretada como consequência dos limites impostos pela capacidade de absorção potencial das firmas. No mesmo sentido, maiores graus de diversificação são observados em firmas de grande porte e com maior capacidade de acumular conhecimentos.

PALAVRAS-CHAVE: diversificação tecnológica; oportunidades tecnológicas; conhecimento e inovação; mudanças climáticas.

CLASSIFICAÇÃO JEL: L25; O33; Q55.

Correspondência para: Paulo Henrique Assis Feitosa

Contato: pfeitosa@usp.br 


\title{
TECHNOLOGICAL OPPORTUNITIES AND FIRMS' DIVERSIFICATION IN CLIMATE CHANGE MITIGATION TECHNOLOGIES
}

\begin{abstract}
This article aims to investigate whether the diversification in climatechange mitigation technologies is affected by changes in technological opportunities at the firm level. From the constitution of a unique database of patents related to climate-change mitigation technologies, this study examines 120 technology groups worldwide and the patent portfolio of 436 firms between 1990 and 2009. The results suggest a non-linear relationship (inverse-U-shape) between the level of technological opportunity and the diversification degree of firms, arguing that the reduction of diversification at a certain point in the curve can be interpreted as a consequence of the limits imposed by the firm's potential absorptive capacity. Similarly, large companies and those with greater capacity to accumulate knowledge show higher levels of technological diversification.
\end{abstract}

KEYWORDS: technological diversification; technological opportunities; knowledge and innovation; climate change. 


\section{INTRODUÇÃO}

A diversificação está no cerne da evolução da empresa moderna, que a utiliza como estratégia de crescimento de longo prazo via expansão para novos mercados geográficos e de produtos. Nessa estratégia, as firmas buscam constantemente capturar novos investimentos lucrativos, motivadas pela possibilidade de eliminar as restrições à sua expansão impostas pela demanda potencial dos produtos que compõem o seu portfólio.

A diversificação é estudada de forma ampla pela literatura e tradicionalmente relacionada a questões como a redução de custos e riscos e a exploração de economias de escala e escopo (TORRISI; GRANSTRAND, 2004). Diante do reconhecimento do crescente papel do conhecimento científico e tecnológico para explicar o crescimento econômico no mundo moderno, diversos autores se dedicaram a compreender a dimensão tecnológica da diversificação corporativa (DOSI; TEECE; CHYTRY, 2004; NELSON; WINTER, 2002; PAVITT; ROBSON; TOWNSEND, 1989). Nesse sentido, a diversificação tecnológica é percebida como uma forma pela qual as firmas criam, ampliam ou modificam a sua coleção de recursos produtivos, que são específicos ao contexto de cada firma e fortemente relacionados às competências e rotinas internas (PENROSE, 1959). A construção dessas capacitações é resultado de processos internos de aprendizado que são experimentados de forma evolucionária em ambientes de seleção.

As causas e os efeitos da diversificação tecnológica são objeto de ampla literatura empírica (BRESCHI; LISSONI; MALERBA, 2003; CORRADINI; DEMIREL; BATTISTI, 2016; LETEN; BELDERBOS; VAN LOOY, 2007). Nesses trabalhos, destaca-se a ênfase conferida às relações entre o nível de diversificação tecnológica e o desempenho inovativo, financeiro e produtivo das firmas, o que resultou na prevalência de análises sobre as variáveis internas da firma. Por outro lado, os estudos que incluem variáveis que controlam os efeitos externos à firma no processo de diversificação tecnológica são menos conclusivos, ainda que se reconheça os avanços empreendidos (CHIU et al., 2010; CORRADINI; DEMIREL; BATTISTI, 2016; DU; LU; GUO, 2015; PATEL; PAVITT, 1997; SEBREK, 2015; TOH; KIM, 2013).

Como consequência, a influência das modificações do ambiente externo no processo de diversificação tecnológica das firmas é um fenômeno que precisa ser mais bem compreendido pela literatura. Diante do fato de que a maioria dos estudos existentes são de natureza transversal e muitas vezes sujeitos a problemas de heterogeneidades não observadas, constata-se a necessidade de compreender como as relações entre diversificação e oportunidade tecnológica se modificam ao longo do tempo. Dessa forma, percebe-se uma lacuna na compreensão de como as firmas, 
de forma mais generalizada, lidam com a diversificação em resposta a rápidas modificações nos níveis de oportunidades tecnológicas.

O primeiro desafio de qualquer estudo que se proponha a lidar com essas limitações é identificar e analisar as transformações no ambiente tecnológico que não sejam específicas a setores industriais, áreas tecnológicas e localidades geográficas. Nesse sentido, os efeitos sociotécnicos das mudanças climáticas constituem um importante caso a ser estudado. A mitigação das emissões de gases de efeito estufa (GEE) é um desafio global, e sua solução exige a reestruturação de ativos tecnológicos constituídos por firmas ao longo de décadas. As tecnologias de mitigação tendem a ser abrangentes, horizontais e pervasivas, características que resultam em modificações nos níveis de oportunidades tecnológicas relacionadas à manufatura, aos meios de distribuição, às redes de serviços e às tecnologias complementares. Ainda que muitas das tecnologias de mitigação sejam reconhecidas por sua especificidade setorial, é importante destacar a sua capacidade de aplicação em diversos domínios, com repercussões socioeconômicas e ambientais em todo o sistema econômico. ${ }^{1}$

O presente artigo tem como objetivo investigar se a diversificação em tecnologias de mitigação das mudanças climáticas é afetada por alterações nos níveis de oportunidades tecnológicas no nível da firma. Para tanto, examina os determinantes da diversificação tecnológica das firmas, com ênfase ao papel exercido pelo custo e pela dificuldade de inovação nesses domínios tecnológicos.

Para tal tarefa, foi constituído um banco de dados original e abrangente com informações de documentos de patentes cuja Classificação Internacional de Patentes (IPC, na sigla em inglês) são considerados pela Organização Mundial de Propriedade Intelectual (OMPI) como Tecnologias Ambientalmente Saudáveis (TAS), conforme listado pela Convenção-Quadro das Nações Unidas sobre Mudança do Clima (CQNUMC). A partir da criação de variáveis e do tratamento econométrico, o artigo analisa o portfólio de 436 firmas, constituído por 95.013 patentes, divididas em 120 grupos de tecnologias, registradas entre os anos de 1990 e 2009.

O artigo está organizado em cinco seções, além desta introdução. A primeira discute os aspectos teóricos e conceituais das oportunidades e diversificação tecnológica. A segunda apresenta a descrição da base de dados construída. A especificação do modelo e suas variáveis são expostas na terceira seção. Os resultados empíricos são apresentados na seção quatro e a última seção apresenta as conclusões.

\footnotetext{
1 Um exemplo dessa pervasividade e abrangência é o subconjunto de tecnologias envolvidas na transição para sistemas energéticos e de transporte com baixa emissão de carbono, que apesar da sua especificidade setorial, produz impactos em uma parcela significativa da economia em escala global.
} 


\section{MUDANÇAS CLIMÁTICAS, OPORTUNIDADES E DIVERSIFICAÇÃO TECNOLÓGICA}

A diversificação corporativa é um fenômeno onipresente no mundo econômico contemporâneo e está intimamente relacionado às mudanças nas condições de oportunidade das empresas. Esse fenômeno é observado pelo grau de dispersão das atividades das firmas em algum intervalo de tempo, e é resultado da busca contínua pelo uso eficiente de recursos produtivos e por meio da captura de oportunidades externas.

Como a produção de novos bens e serviços requer a combinação e integração de uma ampla variedade de conhecimentos tecnológicos, as evidências mostram que a diversificação tecnológica das firmas é geralmente maior do que a diversificação de produtos. Diante da constante incorporação de novas tecnologias, grande parte das empresas pode ser considerada multitecnológica, isto é, que é capaz de dominar um conjunto relativamente amplo de conhecimentos e capacitações, ainda que seja especializada em apenas uma linha de negócios. Esse diagnóstico é corroborado por um fato comum na evolução das indústrias em todo o mundo, que é a prevalência de firmas tecnologicamente diversificadas, ou seja, que cada vez mais inovam e controlam campos tecnológicos diferentes. Essas corporações também registram crescentes níveis de investimento em pesquisa e desenvolvimento $(\mathrm{P} \& \mathrm{D})$, forte articulação externa para o desenvolvimento de novas tecnologias e busca por oportunidades para ingressar em novos mercados a partir do relacionamento de tecnologias (GRANSTRAND; PATEL; PAVITT, 1997; PATEL; PAVITT, 1997).

Os estudos também revelam que o perfil do conhecimento tecnológico das firmas é bastante estável ao longo do tempo. ${ }^{2}$ A direção futura de acumulação tecnológica é restringida pela base de conhecimento mantido pelas firmas no presente, diante do forte componente "dependente da trajetória" (path-dependent) desse processo. Esse conhecimento determina o que a empresa pesquisa e tem capacidade de produzir, dada a existência de limites cognitivos. Além de estável, esse perfil é bastante similar para a maioria das companhias do mesmo segmento industrial, mas fortemente diferenciado entre aquelas que compõem diferentes segmentos. Sabe-se que, para as firmas, individualmente, a diversidade tecnológica garante a base para aperfeiçoar seus produtos. Para a economia como um todo, a diversidade entre as firmas possibilita uma exploração mais completa de mercados de produtos (PATEL; PAVITT, 1997; PAVITT, 1998).

\footnotetext{
2 Um estudo com um conjunto de 440 firmas revela que mais de $90 \%$ tinham perfil tecnológico similar quando comparados os períodos de 1969-1974 e 1985-1990 (PATEL; PAVITT, 1997).
} 
O desenvolvimento de conhecimentos tecnológicos por uma empresa ocorre por meio de um processo de aprendizagem gradual que restringe sua atividade de pesquisa às áreas próximas àquelas que ela já domina. A justificativa principal é o custo para se dominar conhecimentos especializados no mundo contemporâneo, principalmente em grandes firmas, que têm conhecimentos em um vasto número de campos tecnológicos (CANTWELL; FAI, 1999; PAVITT, 1998).

As abordagens sobre a natureza da diversificação tecnológica revelam um fenômeno multifacetado e determinado por questões como: i) o aumento da complexidade dos artefatos e conhecimentos tecnológicos; ii) o acúmulo de conhecimentos fora das competências centrais da firma para dominar as tecnologias subjacentes a seus artefatos; e iii) a base de conhecimentos exigida para aumentar a efetividade da apropriação dos retornos dos esforços de inovação e o lucro esperado (LETEN; BELDERBOS; VAN LOOY, 2007; TORRISI; GRANSTRAND, 2004). Essas análises também mostram que a diversificação não ocorre aleatoriamente e tem certa intencionalidade, em outras palavras, que as firmas têm coerência nas atividades produtivas e tecnológicas que desempenham (SCOTT, 1993; TEECE et al., 1994). Da mesma forma, o interrelacionamento de conhecimento é um fator-chave na explicação da diversificação tecnológica das firmas (BRESCHI; LISSONI; MALERBA, 2003).

A literatura empírica revela a existência de uma relação positiva entre diversificação tecnológica e desempenho econômico (GARCIA-VEGA, 2006; PISCITELLO, 2004), mas também os limites dessa diversificação no seu desempenho, evidenciado pela relação não-linear entre essas variáveis (CHANG et al., 2014; HUANG; CHEN, 2010; LETEN; BELDERBOS; VAN LOOY, 2007). Esse desempenho é medido em termos inovativos, tendo como variável operacional os dados de patentes, mas também em termos financeiros, medido por meio do valor adicionado, retorno de ativos e valor econômico agregado (CHEN; YANG; LIN, 2013).

Em alguns casos, a análise da relação entre diversificação tecnológica e desempenho é realizada a partir da influência de um efeito moderador, que pode ser representado por meio da coerência tecnológica (LETEN; BELDERBOS; VAN LOOY, 2007), dos ativos complementares (LAI et al., 2010), e do excesso de capacidade (organizational slack) das firmas (HUANG; CHEN, 2010).

A partir dessa revisão, é possível perceber uma relativa ênfase das abordagens empíricas à relação entre a diversificação tecnológica e o desempenho das firmas. Entretanto, outros trabalhos têm se empenhado para ampliar os determinantes da diversificação tecnológica, incluindo o papel das oportunidades tecnológicas, o impacto inovativo, o grau de generalidade (CORRADINI; DEMIREL; BATTISTI, 2016), a função de redes sociais externas (DU; LU; GUO, 2015) e os efeitos da diversificação relacionada e não-relacionada (CHEN; CHANG, 2012; CHEN; SHIH; CHANG, 2012). 


\subsection{MUDANÇAS NO AMBIENTE TECNOLÓGICO E NO NÍVEL DE OPORTUNIDADES}

A compreensão dos efeitos das mudanças no ambiente tecnológico, mais especificamente nas oportunidades tecnológicas, é um elemento chave deste artigo. Em geral, os estudos existentes são marcados por limitações, como a dificuldade da generalização dos resultados, uma vez que as abordagens são conduzidas em contextos específicos e recortes setoriais. Outra limitação é a ausência de interpretações de como a relação entre as variáveis muda ao longo do tempo, dado que grande parte dos estudos é de natureza transversal. Como resultado, percebe-se que a influência do ambiente tecnológico no processo de diversificação das firmas é um fenômeno que precisa ser mais bem compreendido pela literatura.

Diante de um conjunto de possibilidades de investigação que permitam a superação dessas limitações, a escolha do processo de mudanças climáticas globais se justifica por uma razão principal: a mitigação das emissões de GEE é um problema que tem, por natureza, uma escala global, ao mesmo tempo que as tecnologias climáticas tendem a ser abrangentes, horizontais e pervasivas. Esses atributos exigem o reposicionamento de estratégia das firmas de todos os tipos e tamanhos, além da reestruturação de ativos tecnológicos constituídos pelas companhias ao longo de décadas (GEELS, 2014). A exploração dessas tecnologias tende a acontecer cada vez mais internacionalmente e está relacionada à emergência de um mercado para tecnologias (ARORA; FOSFURI; GAMBARDELLA, 2001) que criam um amplo conjunto de oportunidades relacionadas à manufatura, aos meios de distribuição, às redes de serviços e às tecnologias complementares. Segundo a Organização para a Cooperação e Desenvolvimento Econômico (OCDE), iluminação, energia elétrica, veículos híbridos e elétricos, geração de energia, baterias e motores são percebidos como as áreas de desenvolvimento mais acelerado de novas tecnologias (OECD, 2014).

De modo geral, a oportunidade tecnológica reflete a facilidade para se inovar a partir de um determinado volume de recursos em pesquisa, e pode ser classificada de acordo com seu nível e pervasividade (CASTELLACCI, 2007; MALERBA; ORSENIGO, 1993). Altos níveis de oportunidade constituem um forte incentivo para a realização de atividades inovadoras, ainda que sejam diferenciadas entre setores e tecnologias. Em alguns setores industriais, essas oportunidades são relacionadas a $\mathrm{P} \& \mathrm{D}$ em equipamentos e instrumentação, enquanto em outras as fontes externas de conhecimento, como fornecedores e clientes, desempenham o papel principal. As condições de oportunidade são dinâmicas por natureza e não se mantêm constantes ou exógenas, pois são geradas e recriadas pela atividade inovativa das firmas. Já a pervasividade das oportunidades é determinada pela capacidade de um novo conhecimento ser aplicado em uma variedade de produtos e mercados. 
Para Klevorick et al. (1995), existem três fontes principais de oportunidades tecnológicas. A primeira é os avanços na compreensão científica que fundamenta toda a atividade de $\mathrm{P} \& \mathrm{D}$ das firmas. Na ciência básica, esses avanços têm impulsionado as taxas de avanço tecnológico em uma ampla gama de setores e têm sido um requisito fundamental para a maioria do progresso tecnológico do último século. A segunda fonte é o progresso tecnológico que tem origem em outras indústrias, ou seja, as oportunidades tecnológicas surgem dos avanços tecnológicos no restante da economia. A terceira fonte se relaciona à retroalimentação (feedbacks), pois em muitos setores industriais a pesquisa que é realizada no presente gera novos conhecimentos e aumenta as oportunidades tecnológicas futuras.

O perfil tecnológico das firmas se modifica lentamente ao longo do tempo, em resposta a mudanças nas oportunidades tecnológicas. Como resultado, em áreas com altas oportunidades tecnológicas as firmas tendem a se transformar em multiproduto, bem como multitecnologia (PAVITT, 1998). O dinamismo ambiental é um fator que limita as vantagens associadas a competências específicas das firmas (LIN; CHANG, 2015). Ao mesmo tempo, os diferentes níveis de dinamismo do ambiente, que se aplicam em diferentes contextos, tornam as competências tecnológicas obsoletas mais rapidamente em alguns setores em detrimento de outros.

O nível de oportunidades tecnológica também está intimamente relacionado à capacidade de absorção das firmas, ou seja, ao alcance da sua aptidão em reconhecer o valor de novas informações, assimilá-las e aplicá-las para fins comerciais (COHEN; LEVINTHAL, 1990). Dessa forma, enquanto a capacidade de absorção potencial se refere à capacidade das firmas de adquirir e assimilar conhecimento gerado externamente, a capacidade realizada está associada à forma como transformam e exploraram esse conhecimento para fins comerciais (ZAHRA; GEORGE, 2002). As firmas se diferem em sua capacidade de criar valor a partir da sua capacidade de absorção, e nem toda aquisição e assimilação de novos conhecimentos resulta numa exploração comercial bem sucedida (JANSEN; VAN DEN BOSCH; VOLBERDA, 2005).

\section{DESCRIÇÃO DA BASE DE DADOS}

A base de dados utilizada neste artigo tem como fonte de informação o Banco Mundial de Estatísticas de Patentes (PATSTAT) do European Patent Office (EPO), em sua edição de abril de 2012. Essa base de dados abrange mais de 80 escritórios de propriedade intelectual em todo o mundo e contém mais de 70 milhões de documentos de patentes. Adicionalmente, também é utilizado o banco de dados comercial ORBIS da Bureau Van Dijck (BvDEP), que contém informações de 120 milhões de empresas ou registros de negócios ao redor do mundo (dado de outubro de 2015). 
A construção da base de dados envolveu a extração e o tratamento das informações em três etapas principais. A primeira consiste na seleção das "patentes verdes". A partir do banco de dados do EPO/PATSTAT, foram selecionados todos os depósitos de patentes com um ou mais IPC classificados no Green Inventory ${ }^{3}$ como Tecnologias Ambientalmente Saudáveis (TAS). Partindo de um universo de aproximadamente 73 milhões de patentes em todo o mundo, foram selecionados 5.297.210 depósitos denominados como "patentes verdes". Ressalta-se a abrangência dos escritórios analisados que, ao contrário de estudos prévios que se concentraram em áreas geográficas mais delimitadas ou em setores específicos (CORRADINI; DEMIREL; BATTISTI, 2016), permite uma perspectiva de análise do fenômeno para além de setores industriais e recortes geográficos e tecnológicos.

Para o tratamento de agregados internacionais comparáveis, o artigo utiliza informações das famílias de patentes. Como existem diversas formas para estabelecer a relação entre os documentos de patentes para a formação de uma família, adota-se um método que consiste em identificar as patentes prioritárias transnacionais (PPT). Esse método tem sido utilizado em diversos estudos sobre o tema (ALKEMADE et al., 2015; DE RASSENFOSSE et al., 2013; PICCI; SAVORELLI, 2012) e usufrui da possibilidade de o PATSTAT fornecer dados originais das prioridades de todos os depósitos de patentes. Na segunda etapa, a partir da aplicação do primeiro filtro, foram identificadas 2.535 .867 famílias de patentes que foram relacionadas a 764.668 patentes prioritárias.

Para se chegar à terceira etapa foram aplicados outros dois filtros; o filtro 2 faz um recorte temporal de vinte anos (1990-2009), tendo como referência os dados mais recentes permitidos pela base utilizada. ${ }^{4}$ Esse recorte foi distribuído em períodos de cinco anos e se justifica por representar uma janela temporal adequada para avaliar o impacto tecnológico (HENDERSON; COCKBURN, 1996; NOOTEBOOM et al., 2007; PODOLNY; STUART, 1995), além de estar relacionado ao tempo médio de exame dos pedidos de patentes em escritórios em todo o mundo (DE RASSENFOSSE; PALANGKARAYA; WEBSTER, 2016). O filtro 3 seleciona as firmas que nesse período depositaram, em média, mais do que duas patentes por ano. Esse critério é relevante para que a amostra revele apenas os depositantes com uma sistemática e coerente atividade inovativa nessas tecnologias. Nessa etapa também foram

3 Classificação desenvolvida pela Organização Mundial de Propriedade Intelectual (OMPI) que divide as tecnologias numa estrutura hierárquica, distribuída em setores e subsetores.

4 Para a produção de indicadores representativos, os documentos de referência em estatística de patentes sugerem um mínimo de três anos entre a sistematização de dados dos escritórios e a seleção dos depósitos de patentes (OECD, 2009). Como o presente artigo utiliza a edição de abril de 2012 do EPO/PATSTAT, a atualidade máxima permitida é o ano de 2009. 
excluídos inventores individuais, universidades e institutos de pesquisa. O diagrama de fluxo que sintetiza o processo de extração e o tratamento das informações nas três etapas é apresentado na Figura 1.

Figura 1 - Diagrama de fluxo do processo de extração e tratamento dos dados
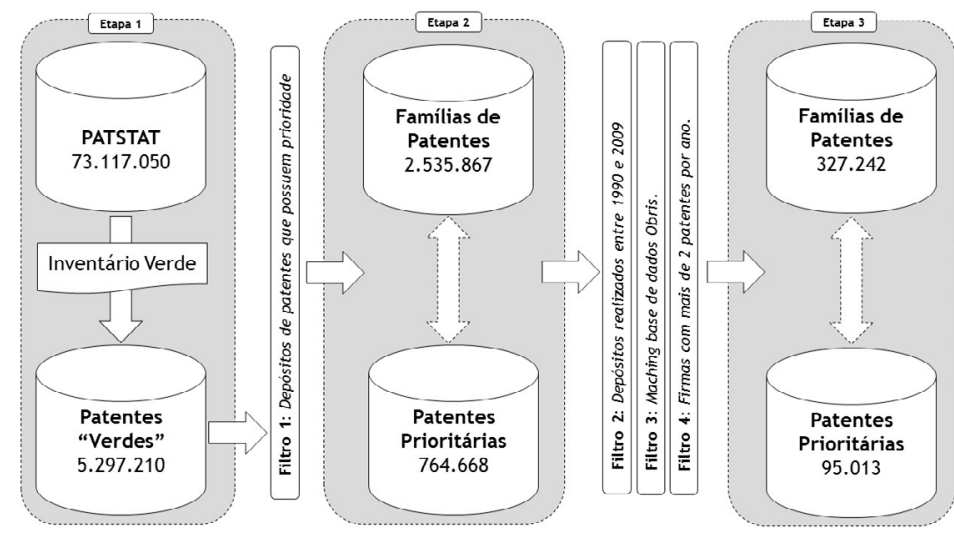

Fonte: Elaboração própria.

O processo de extração de tratamento de dados resultou em 95.013 depósitos de patentes realizados por 436 firmas em 37 escritórios de todo o mundo. Por fim, associa-se as informações dos primeiros depositantes de todas as patentes a informações da base de dados do Orbis. Essa tarefa tem como objetivo obter informações adicionais sobre as companhias, principalmente seus tamanhos, medidos em termos do total de ativos.

\section{ABORDAGEM EMPÍRICA}

A mensuração da diversificação tecnológica é central para a proposta empírica deste artigo. Para tanto, ela é entendida como o grau de dispersão de atividades ou resultados que se define $X$, numa determinada unidade analítica $Y$. Nesses termos, $X$ pode se referir a um conjunto de produtos, serviços ou mercados, enquanto a unidade $Y$ pode ser uma firma, indústria, país, região ou um indivíduo.

Tradicionalmente, a literatura examina a diversificação em termos de produtos ou mercados. Este artigo se soma aos esforços que complementam essas formas de diversificação e incluem outros insumos usados na produção, como tecnologias, conhecimentos, competências, relações de rede e outras formas de recursos intangíveis (CANTWELL; GAMBARDELLA; GRANSTRAND, 2004; TORRISI; GRANSTRAND, 2004). 
Neste artigo, $Y$ são unidades organizacionais e $X$ representam suas competências, interpretadas por classes tecnológicas. Em estudos baseados em estatísticas de patentes, a medida mais usual e precisa de diversidade tecnológica é a quantidade e proporção dos campos técnicos em que as firmas depositam patentes e, por consequência, têm competências tecnológicas. Dibiaggio e Nesta (2005) afirmam que as classes tecnológicas podem ser consideradas as unidades de análise mais apropriadas para explorar as informações contidas nas bases de dados de patentes.

Entretanto, essas medidas precisam ser vistas com cautela, pois é precipitado restringir as competências de uma corporação em termos de alguns campos de excelência. Em vez disso, é mais útil pensar em termos de perfis de competências, com diferentes níveis de vantagem competitiva em uma variedade de campos tecnológicos (PATEL; PAVITT, 1997). Dado que o atributo da diversificação tecnológica da firma consiste na mensuração de quotas de um portfólio tecnológico, as medidas mais comuns nos trabalhos que estimam essa variável a partir de dados de patentes são baseadas no indicador de entropia e no indicador Herfindahl-Hirschman (HH).

A medida baseada no Herfindahl-Hirschman, convencionalmente utilizada para dimensionar as firmas com relação à sua indústria e ao grau de concorrência, se tornou um indicador muito utilizado em estudos sobre diversificação corporativa (BERRY, 1971; MONTGOMERY, 1982) e na mensuração da diversificação tecnológica (CORRADINI; DE PROPRIS, 2016; CORRADINI; DEMIREL; BATTISTI, 2016; GARCIA-VEGA, 2006; KRAMMER, 2016; LETEN; BELDERBOS; VAN LOOY, 2007; LIN; CHANG, 2015; QUINTANA-GARCÍA; BENAVIDES-VELASCO, 2008).

Em termos formais, o indicador captura a dispersão do portfólio de patentes das firmas em relação aos campos tecnológicos nos quais ela atua. Para tanto, o indicador é estimado pelo cálculo do complemento do índice HerfindahlHirschman, confrontando o número de patentes para cada IPC com o número total de patentes da empresa $i$ para cada período (ou quinquênio) $t$, o que pode ser expresso da seguinte forma:

$$
\text { TECHDIV } i t=\frac{N_{i t}}{N_{i t}-1}\left(1-\sum_{k=1}^{K}\left(\frac{N_{i t, k}}{N_{i t}}\right)^{2}\right)
$$

Em (1), $N_{i t}$ é o número total de patentes para a empresa $i$ para cada período (ou quinquênio) $t$, enquanto $k$ representa a IPC em que a empresa patenteou e $K$ é o número total de classes tecnológicas em que a empresa é ativa. Como indicado por Hall (2005) e aplicado em estudos similares (CORRADINI; DE PROPRIS, 2016; CORRADINI; DEMIREL; BATTISTI, 2016; CORROCHER; MALERBA; MONTOBBIO, 2007; RAHKO, 
2016), esse indicador é normalizado $\left(N_{i t} / N_{i t}-1\right)$ para corrigir o problema de viés em observações com poucas patentes por ano.

A revisão de literatura sobre o uso desses indicadores revela que o $\mathrm{HH}$ é mais frequentemente utilizado em estudos empíricos sobre diversificação tecnológica. Ademais, é preciso reconhecer algumas limitações dessa mensuração, como nos casos em que a atividade tecnológica da firma se encontra em estágio embrionário e, por consequência, é de pequena escala. Nessas circunstâncias, as estimativas podem subestimar a diversificação das firmas, ainda que esse não seja o caso das firmas representadas neste artigo (CHEN; JANG; WEN, 2010).

A adoção do indicador $\mathrm{HH}$ tem três justificativas principais. Em primeiro, a tentativa de tornar seus resultados passíveis de comparação com estudos similares sobre o tema. Em segundo, o HH apresenta-se mais sensível e adequado à análise de grandes portfólios e ao número elevado de classes tecnológicas, como é o caso deste trabalho. E em terceiro, as estimativas realizadas com um indicador de entropia, a principal alternativa ao $\mathrm{HH}$, mostraram que as diferenças são pequenas e não interferem de forma significativa nos resultados alcançados.

\subsection{VARIÁVEIS INDEPENDENTES}

Outra medida fundamental para a proposta empírica deste trabalho advém da noção de oportunidade tecnológica. Existe ampla aceitação entre os estudiosos do fato de que indústrias diferem no nível de oportunidades que enfrentam para conduzir o avanço técnico. Por outro lado, não há consenso sobre como tornar o conceito de oportunidade tecnológica preciso e empiricamente operacionalizado (CASTELLACCI, 2007; COHEN; LEVIN, 1989). Dessa forma, as investigações sobre oportunidades tecnológicas sofrem dificuldade em estabelecer uma definição operacional desse conceito que permita alguma forma de mensuração (KLEVORICK et al., 1995)

Normalmente, a medida de oportunidade tecnológica baseia-se no aumento da taxa de atividade inovativa nas classes tecnológicas que a firma opera (MALERBA; ORSENIGO, 1993). Neste trabalho, a medida proposta é inspirada em Patel e Pavitt (1998), também aplicada em estudos posteriores (CORRADINI; DEMIREL; BATTISTI, 2016), e consiste na taxa de crescimento geométrico anual do número de patentes para cada classe tecnológica (OPPOR). Essa variável é calculada para cada firma, pois pressupõe que o conjunto de oportunidades para investimento e crescimento percebidas por empresários e administradores é específico de cada firma, como sugere Penrose (1959). Para testar a não linearidade entre essas variáveis, também é estimada uma função quadrática da variável oportunidade tecnológica por meio do seu termo ao quadrado $\left(\mathrm{OPPOR}^{2}\right)$. 
Para controlar os efeitos de diferentes competências e capacitações das firmas, também se estima seu estoque de conhecimento por meio do número de patentes acumulado em períodos prévios (KSTOCK). A construção dessa variável é originalmente inspirada na função de acumulação de Popp (2012), mas excluindo-se o componente de difusão, como proposto por Costantini e Mazzanti (2012). Assim como convencionado pela literatura, essa variável considera uma taxa de depreciação de 15\% (HALL; JAFFE; TRAJTENBERG, 2005). Já a influência do tamanho das firmas é estimada pela variável (SIZE), inspirada por Nesta e Saviotti (2005) e construída a partir das informações do seu total de ativos, extraídas do banco de dados da Orbis. Em ambos os casos, as variáveis são introduzidas no modelo após serem transformadas em escalas logarítmicas.

\subsection{ESTATÍSTICAS DESCRITIVAS}

Tendo como ponto de partida a identificação de depósitos de patentes com campos técnicos relacionados a tecnologias de mitigação das mudanças climáticas, o trabalho mapeia as 436 firmas com sistemática e coerente atividade inventiva nessa área. Essas companhias representam um conjunto heterogêneo em diversos aspectos. Estão geograficamente distribuídas em 23 países - a destacar o Japão, com 30\% do total, os Estados Unidos, com 26\%, e a Alemanha, com 16\% - e suas atividades econômicas principais estão distribuídas em 116 áreas, de acordo com a classificação estatística de atividades econômicas da Comunidade Europeia (NACE Rev. 2.0).

Tabela 1 - Estatísticas descritivas das variáveis utilizadas no modelo

\begin{tabular}{clcccc}
\hline & \multicolumn{1}{c}{ Descrição } & Média & $\begin{array}{c}\text { Desvio } \\
\text { padrão }\end{array}$ & Mínimo & Máximo \\
\hline \multirow{2}{*}{ TECHDIV } & $\begin{array}{l}\text { Indicador de diversificação tecnológica - dispersão do } \\
\text { portfólio de patentes entre classes }\end{array}$ & 0,5400 & 0,2953 & 0 & 1 \\
& $\begin{array}{l}\text { Variável binária construída a partir do indicador } \\
\text { TECHDIV_B } \\
\text { TECHDIV para estimação dos modelos logit }\end{array}$ & 0,8940 & 0,3478 & 0 & 1 \\
OPPOR & $\begin{array}{l}\text { Condições de oportunidade - taxa de mudança setorial } \\
\text { na atividade de patenteamento }\end{array}$ & 0,5040 & 0,4067 & $-0,5075$ & 2,9644 \\
KSTOCK & $\begin{array}{l}\text { Estoque de conhecimento da firma - Logaritmo do total } \\
\text { de patentes acumuladas pela firma em períodos prévios }\end{array}$ & 3,7856 & 1,3807 & 0,5306 & 8,0559 \\
& $\begin{array}{l}\text { Tamanho da firma - Logaritmo do total de ativos da } \\
\text { firma em US\$ }\end{array}$ & 15,4038 & 2,6259 & 2,7725 & 19,8709 \\
\hline
\end{tabular}

Nota: As variáveis foram estimadas a partir de EPO (2012), exceto a variável SIZE, produzida a partir de Bureau Van Dijk (2013). Fonte: Elaboração própria.

A Tabela 1 descreve as principais estatísticas descritivas das variáveis que são utilizadas no modelo de regressão. Esses resultados mostram que existe uma importante 
diferença entre as firmas no que se refere ao seu nível de diversificação tecnológica, o que também reflete o grande escopo de áreas técnicas analisadas. Do mesmo modo, as firmas se diferenciam no seu nível de oportunidades tecnológicas, ou seja, na sua capacidade de capturar essas oportunidades entre tecnologias ao longo do tempo.

\subsection{ESTIMAÇÃO DO MODELO}

O primeiro problema a ser enfrentado nas estimativas propostas é a distribuição não normal dos dados e a natureza restrita da medida de diversificação tecnológica, que varia no intervalo entre 0 e 1. Como sugerem Papke e Wooldridge (1996), essas características podem levar a valores presumidos numa regressão com mínimos quadrados ordinários (MQO). A alternativa de modelar o $\log$ da razão de chances (log-odds ratio) como uma função linear é inadequada, uma vez que não pode lidar com os casos em que a variável dependente é igual aos limites do intervalo de 0 e 1 . Da mesma forma, o ajustamento de valores extremos também é de difícil justificativa quando estes representam uma grande porcentagem dos dados.

A medida de diversificação tecnológica das firmas resulta numa variável fracionária, assim como diversas outras variáveis possíveis de identificação em contextos econômicos. Para tratar adequadamente desse tipo de variável, o trabalho se inspirou em Papke e Wooldrige (1996), que ampliam a literatura sobre modelos lineares generalizados (MLG) e quase-verossimilhança, propondo um modelo de resposta para obter métodos robustos para estimação e inferência com variáveis de resposta fracionária. De acordo com os autores, esse modelo parte do pressuposto de que a expectativa condicional da variável de resposta fracionária é definida como:

$$
E\left(y_{i} \mid x_{i}\right)=G\left(x_{i} \beta\right)
$$

Em (2), $i=1,2, \ldots, N$ e $G$ (.) é uma função de distribuição acumulada, tal qual a função logística $G(z) \equiv \Lambda(\mathrm{z}) \equiv \exp (z) /(1+\exp (z))$, que satisfaz $0<G(z)<1$ para todo $z \in \mathbb{R}$ e garante que os valores previstos de $y$ estão no intervalo $(0,1)$. Seguindo Papke e Wooldrige (1996), é então possível maximizar uma função de probabilidade logarítmica de Bernoulli expressa como:

$$
l_{i}(\beta)=y_{i} \log \left[G\left(x_{i} \beta\right)\right]+\left(1-y_{1}\right) \log \left[1-G\left(x_{i} \beta\right)\right]
$$

Essa função permite obter um estimador de máxima quase-verossimilhança de $\beta$ que é consistente independentemente da distribuição $y_{i}$ condicional a $x_{i}$ usando regressões logit ou probit. Assim, dada a estrutura não-balanceada dos dados e o número limitado de observações por painel, os dados foram organizados pressupondo o potencial dos erros-padrão robustos em explicar heterogeneidades e a dependência estatística das séries ao longo do tempo. 


\section{ANÁLISE DOS RESULTADOS}

Os resultados das estimativas econométricas da relação entre oportunidade e diversificação tecnológica estão na Tabela 2. A primeira e a segunda coluna apresentam a estimação linear; a terceira e a quarta mostram o modelo fracionário; e a quinta coluna, o modelo logit. A estimação de diferentes modelos tem como objetivo estabelecer uma linha base a partir de estruturas lineares (colunas 1 e 2) para, em seguida, orientar as estimativas que sustentam a estratégia empírica (colunas 3, 4 e 5). A partir dessa divisão, espera-se interpretar adequadamente o fenômeno investigado e lidar com os desafios impostos pela natureza dos dados. Nos dois primeiros casos, os exercícios econométricos se iniciam com a inclusão das variáveis lineares e quadrática da oportunidade tecnológica, juntamente com as variáveis de controle, para em seguida incluir as variáveis estoque de conhecimento e tamanho das firmas. Na quinta coluna, estima-se um modelo logístico de máxima verossimilhança padrão. Nesse caso, converte-se a variável dependente em uma variável binária (TECHDIV_B), que é igual a 1 para todas as observações maiores que zero. A inclusão do modelo logit padrão tem fins apenas comparativos, sendo que é preciso reconhecer os problemas de perda de informações na conversão da variável dependente em uma variável binária.

\begin{tabular}{|c|c|c|c|c|c|}
\hline \multirow{3}{*}{$\begin{array}{c}\text { Variável dependente } \\
\text { Modelo } \\
\text { Variáveis independentes/controle }\end{array}$} & \multicolumn{5}{|c|}{ TECHDIV } \\
\hline & \multicolumn{2}{|c|}{ Linear } & \multicolumn{2}{|c|}{ Logit fracionária $a^{5}$} & \multirow{2}{*}{$\frac{\text { Logit }}{(5)}$} \\
\hline & (1) & (2) & (3) & (4) & \\
\hline OPPOR & $\begin{array}{l}0,2282^{\star * *} \\
(0,0463)\end{array}$ & $\begin{array}{l}0,1699^{* * *} \\
(0,0516)\end{array}$ & $\begin{array}{l}0,9425^{\star * *} \\
(0,2007)\end{array}$ & $\begin{array}{l}0,7261^{\star * *} \\
(0,2244)\end{array}$ & $\begin{array}{l}2,2267^{* * *} \\
(0,6182)\end{array}$ \\
\hline $\mathrm{OPPOR}^{2}$ & $\begin{array}{l}-0,1190^{* * *} \\
(0,0285)\end{array}$ & $\begin{array}{l}-0,0883^{* * *} \\
(0,0305)\end{array}$ & $\begin{array}{l}-0,4960^{\star * *} \\
(0,1289)\end{array}$ & $\begin{array}{l}-0,3796^{* * *} \\
(0,1363)\end{array}$ & $\begin{array}{l}-0,3999 \\
(0,4039)\end{array}$ \\
\hline KSTOCK & & $\begin{array}{l}0,0568^{\star *} \\
(0,0058)\end{array}$ & & $\begin{array}{l}0,0822^{\star * *} \\
(0,0311)\end{array}$ & $\begin{array}{l}0,8077^{* * *} \\
(0,1136)\end{array}$ \\
\hline SIZE & & $\begin{array}{l}0,0457^{\star * *} \\
(0,0051)\end{array}$ & & $\begin{array}{l}0,2425^{\star * *} \\
(0,0257)\end{array}$ & $\begin{array}{l}0,1652^{* *} \\
(0,0368)\end{array}$ \\
\hline Constante & $\begin{array}{l}0,4476^{* * *} \\
(0,0950)\end{array}$ & $\begin{array}{l}-0,4879^{* * *} \\
(0,1329)\end{array}$ & $\begin{array}{l}-0,2181 \\
(0,3844)\end{array}$ & $\begin{array}{l}-4,2139^{* * *} \\
(0,5603)\end{array}$ & $\begin{array}{l}-2,8385^{* * *} \\
(0,6716)\end{array}$ \\
\hline Dummies períodos & $\operatorname{Sim}$ & $\operatorname{Sim}$ & Sim & $\operatorname{Sim}$ & Sim \\
\hline Dummies tamanho da firma & Sim & Sim & Sim & Sim & Não \\
\hline Número de firmas & 436 & 301 & 436 & 301 & 301 \\
\hline Número de observações & 1473 & 1019 & 1473 & 1019 & 1019 \\
\hline Log pseudo-verossimilhança & & & $-773,01$ & $-508,80$ & $-210,38$ \\
\hline AIC & & & 1,0631 & 1,0221 & \\
\hline
\end{tabular}

Nota: ${ }^{\star} p<0,10 ;{ }^{* *} p<0,05 ;{ }^{* *} p<0,01$. Erro-padrão robusto em parênteses.

Fonte: Elaboração própria com base em dados de EPO (2012) e Bureau Van Dijk (2013)

\footnotetext{
5 Sobre a estimação do modelo Logit fracionário por meio do software Stata, vide Baum (2008).
} 
Em todos os modelos, o coeficiente da variável oportunidade tecnológica (OPPOR) é positivo e estatisticamente significativo, e o coeficiente da variável quadrática da oportunidade tecnológica $\left(\mathrm{OPPOR}^{2}\right)$ é negativo e significante. Esses resultados representam evidências importantes da existência de uma relação não linear em formato de " $U$ " invertido entre oportunidade e diversificação tecnológica. No nível da firma, é possível interpretar que maiores níveis de diversificação estão positivamente relacionados ao aumento das condições de oportunidade observadas. Entretanto, como a relação assume a forma de "U" invertido, o aumento da diversificação ocorre a uma taxa decrescente até atingir um ponto máximo, o que sugere a existência de um nível ótimo de diversificação tecnológica da firma. A partir desse nível, aumentos da diversificação tendem a exercer impacto negativo sobre o seu grau de inventividade.

Estudos prévios mostram resultados semelhantes no que se refere ao efeito do contexto ambiental nas estratégias de diversificação tecnológica das firmas (CHIU et al., 2010; SEBREK, 2015) e à redução da diversificação como consequência de ambientes com elevada incerteza tecnológica (CORRADINI; DEMIREL; BATTISTI, 2016; TOH; KIM, 2013). Porém, a redução da diversificação a partir de determinado ponto da curva é interpretada, mais especificamente, como consequência dos limites impostos pela capacidade de absorção potencial das firmas. Assim, diferente da capacidade de absorção realizada, que está relacionada à transformação e exploração para fins comerciais, a potencial tende a impor um limite à capacidade das firmas de adquirir e assimilar conhecimento gerado externamente, independentemente do nível de oportunidade tecnológica.

Outro resultado alcançado é que a diversificação tecnológica relacionada à mitigação das mudanças climáticas é positivamente afetada pelo tamanho da firma e por seus estoques de conhecimento. Esses resultados confirmam a hipótese inicial e são estatisticamente significativos na maioria das regressões realizadas, mostrando que as empresas alcançam maiores níveis de diversificação nesse campo quanto maior for a dimensão de seus ativos, mas principalmente de ativos intangíveis, que podem ser representados pelo acúmulo de conhecimentos. Dessa maneira, firmas com diferentes estruturas e tamanhos são afetadas de forma distinta pela limitação de recursos existentes, e esse efeito parece ser mais evidente em firmas de pequeno porte, como constataram Corradini, Demirel e Battisti (2016). Já as grandes firmas de multitecnologia, para lidar com problemas advindos do crescimento do escopo de atividades, utilizam sua estrutura multidivisional para gerenciar cada tecnologia de acordo com suas características de funcionamento e necessidades (LAI et al., 2010).

Nas estimativas seguintes, o artigo procura controlar as heterogeneidades individuais e explorar o máximo potencial da disposição dos dados em painel. Como foi discutido, essa iniciativa parte do diagnóstico de que os estudos prévios, por serem transversais, estão sujeitos a problemas de heterogeneidade não observada. Dessa forma, busca-se aqui extrapolar essas limitações, colocando a dimensão temporal no centro da análise. 
A variável oportunidade tecnológica (OPPOR) controla o comportamento individual, tanto entre as firmas quanto ao longo do tempo. Esse painel é por natureza não balanceado, pois nem todas as varáveis estão disponíveis para todas as firmas em todos os períodos. É também do tipo curto, dado o grande número de firmas e o pequeno número de períodos analisados. A Tabela 3 sintetiza os resultados das variâncias estudadas, mostrando os efeitos between e within.

Tabela 3 - Estimativa das variâncias estudadas

\begin{tabular}{cccc}
\hline Variável & Geral & Between & Within \\
\hline TECHDIV & 0,2953 & 0,2509 & 0,1600 \\
OPPOR & 0,4067 & 0,2613 & 0,3438 \\
OPPOR2 & 0,6812 & 0,5326 & 0,5470 \\
KSTOCK & 1,3807 & 0,9682 & 0,9561 \\
SIZE & 2,6259 & 2,6541 & 0,0000 \\
\hline
\end{tabular}

Fonte: Elaboração própria.

Esses resultados revelam importantes padrões de evolução nas variáveis diversificação e oportunidade tecnológica. No que se refere à diversificação tecnológica, percebe-se que a variância é maior entre as firmas (between) do que se comparada à variância entre os períodos (within). Esse resultado pode ser visto como consequência tanto da relativa estabilidade da diversificação ao longo do tempo quanto da forte heterogeneidade entre as firmas no que se refere ao seu perfil tecnológico. Essas evidências ratificam os estudos prévios que mostram que o perfil tecnológico das firmas é fortemente diferenciado, mas também relativamente estável ao longo do tempo (PATEL; PAVITT, 1997). Como resultado, é possível afirmar que existe um certo padrão temporal no grau de diversificação das firmas.

Ao analisar essas variâncias a partir da variável oportunidade tecnológica, percebe-se o resultado inverso. Nesse caso, a variância entre os períodos (within) é superior à variância entre as firmas (between), o que pode revelar importantes transformações nos níveis de oportunidade tecnológica ao longo do tempo nessas tecnologias. Dessa forma, é possível constatar que o custo e a dificuldade de inovar nessas tecnologias variam relativamente menos entre as firmas, independentemente do seu tamanho, setor econômico ou localização.

As estimativas econométricas dos coeficientes principais com efeitos fixos e aleatórios estão apresentadas na Tabela 4. Nessas estimativas, procura-se tornar os resultados mais robustos e explorar o potencial da análise dos dados em painel para identificar mudanças estruturais na relação entre as variáveis. A primeira e a segunda coluna apresentam a estimação com o modelo com efeitos fixos, e a terceira e a quarta o modelo com efeitos aleatórios. Assim como nas regressões anteriores, os exercícios econométricos iniciam com a inclusão das variáveis lineares e quadrática da 
oportunidade tecnológica, juntamente com as variáveis de controle, para em seguida incluir a variável estoque de conhecimento e tamanho das firmas.

\section{Tabela 4 - Estimativa dos coeficientes das regressões em modelos between, efeitos fixos e aleatórios}

\begin{tabular}{|c|c|c|c|c|c|c|}
\hline \multirow{3}{*}{$\begin{array}{c}\text { Variável dependente } \\
\text { Modelo } \\
\begin{array}{c}\text { Variáveis independentes/ } \\
\text { controle }\end{array}\end{array}$} & \multicolumn{4}{|c|}{ TECHDIV } & & \\
\hline & \multicolumn{2}{|c|}{ Between } & \multicolumn{2}{|c|}{ Efeitos fixos } & \multicolumn{2}{|c|}{ Efeitos aleatórios } \\
\hline & (1) & (2) & (3) & (4) & (5) & (5) \\
\hline OPPOR & $\begin{array}{c}0,3260^{* * *} \\
(0,1069)\end{array}$ & $\begin{array}{l}0,2397^{*} \\
(0,1271)\end{array}$ & $\begin{array}{c}0,1504^{* * *} \\
(0,0368)\end{array}$ & $\begin{array}{l}0,0794^{* *} \\
(0,0331)\end{array}$ & $\begin{array}{c}0,1622^{* * *} \\
(0,0359)\end{array}$ & $\begin{array}{l}0,0953^{* *} \\
(0,0388)\end{array}$ \\
\hline $\mathrm{OPPOR}^{2}$ & $\begin{array}{c}-0,1435^{\star * *} \\
(0,0495)\end{array}$ & $\begin{array}{c}-0,1286^{\star *} \\
(0,0545)\end{array}$ & $\begin{array}{c}-0,0677^{* * *} \\
(0,0201)\end{array}$ & $\begin{array}{c}-0,0415^{\star *} \\
(0,0179)\end{array}$ & $\begin{array}{c}-0,0766^{\star * *} \\
(0,0199)\end{array}$ & $\begin{array}{c}-0,0401^{\star *} \\
(0,0201)\end{array}$ \\
\hline KSTOCK & & $\begin{array}{c}0,0168 \\
(0,0145)\end{array}$ & & $\begin{array}{c}0,0167 \\
(0,0128)\end{array}$ & & $\begin{array}{c}0,0104 \\
(0,0096)\end{array}$ \\
\hline SIZE & & $\begin{array}{c}0,0577^{\star * *} \\
(0,0086)\end{array}$ & & & & $\begin{array}{c}0,0604^{\star * *} \\
(0,0088)\end{array}$ \\
\hline Constante & $\begin{array}{c}0,6921^{* * *} \\
(0,1939)\end{array}$ & $\begin{array}{l}-0,4064 \\
(0,2802)\end{array}$ & $\begin{array}{c}0,4577^{* * *} \\
(0,0109)\end{array}$ & $\begin{array}{c}0,4675 \\
(0,0351)\end{array}$ & $\begin{array}{c}0,4678^{* * *} \\
(00869)\end{array}$ & $\begin{array}{c}-0,4996^{* * *} \\
(0,1216)\end{array}$ \\
\hline Dummies períodos & Sim & Sim & Sim & Sim & Sim & Sim \\
\hline Dummies tamanho & Sim & Sim & Não & Não & Sim & Sim \\
\hline Número de firmas & 436 & 301 & 436 & 436 & 436 & 301 \\
\hline Número de observações & 1473 & 1019 & 1473 & 1443 & 1473 & 1019 \\
\hline
\end{tabular}

Notas: ${ }^{\star} p<0,10 ;{ }^{*} p<0,05 ;{ }^{* *} p<0,01$. Erro-padrão robusto em parênteses.

Fonte: Elaboração própria com base em dados de EPO (2012) e Bureau Van Dijk (2013).

Para todos os modelos propostos, a variável oportunidade tecnológica é mais uma vez estatisticamente significativa, e em todos os casos o coeficiente associado à variável OPPOR é positivo e o coeficiente associado a OPPOR ${ }^{2}$ é negativo, como nas estimativas anteriores. Esses resultados reforçam as evidências da presença de uma relação não linear em formato de " $U$ " invertido entre oportunidade e diversificação tecnológica.

Os resultados encontrados têm importantes repercussões para a literatura. Uma delas está em se somar aos estudos que procuram ampliar a análise dos determinantes da diversificação tecnológica no nível da firma para além de variáveis de desempenho econômico convencionais. Mais especificamente, estudos que visam melhorar a compreensão de como a diversificação é afetada pelo nível de oportunidade tecnológica. Sobre o objeto estudado, uma implicação é demonstrar que os investimentos nessas tecnologias constituem oportunidades efetivas de aumento da performance das firmas e para a criação de mercados em escala interacional. Ainda que a avaliação qualitativa desse processo de difusão esteja além dos objetivos deste artigo, é evidente a sua contribuição para se alcançar uma economia de baixa emissão de carbono. 
Como foi discutido, as variáveis diversificação e oportunidade tecnológica estão presentes em diversos estudos e relacionadas a um conjunto variado de fenômenos. Entretanto, nenhum deles analisou sistematicamente as relações empíricas entre essas variáveis e suas repercussões para a literatura, o que garante a originalidade da abordagem e de seus resultados, dada a abrangência e a forma como essas variáveis são relacionadas.

\section{CONSIDERAÇÕES FINAIS}

Este artigo teve como objetivo investigar se a diversificação em tecnologias de mitigação das mudanças climáticas é afetada por alterações nos níveis de oportunidades tecnológicas no nível da firma. Para tanto, foi constituído um banco de dados original com patentes relacionadas a tecnologias de mitigação das mudanças climáticas, possibilitando a análise de 120 grupos de tecnologias em todo o mundo e da evolução do portfólio de patentes de 436 firmas entre os anos 1990-2009. As estimativas econométricas oferecem evidências empíricas originais sobre a natureza e determinantes da diversificação tecnológica no que se refere ao papel exercido por mudanças no ambiente tecnológico. Os resultados revelam uma relação não linear e em formato de "U" invertido entre oportunidade e diversificação tecnológica.

$\mathrm{O}$ artigo revela de forma abrangente o papel que o ambiente tecnológico exerce sobre a atividade inovativa das firmas, bem como o impacto sobre o grau de diversificação tecnológica. Os resultados ratificam estudos prévios que mostram que o perfil tecnológico das firmas é fortemente diferenciado, mas também relativamente estável ao longo do tempo.

Esse resultado preenche uma lacuna dos estudos empíricos que, em sua maioria, são realizados a partir de análises transversais e passíveis de problemas relacionados a heterogeneidades não observadas. Sobre a interpretação da taxa decrescente da diversificação a partir de determinado ponto da curva, este artigo argumenta que se trata dos limites impostos pela capacidade de absorção potencial das firmas. Todavia, é possível perceber que esses padrões são alterados ao longo do tempo e revelam um comportamento adaptativo das firmas ao seu contexto.

As possíveis explicações para esse comportamento são a exigência de capacitações tecnológicas avançadas para a exploração dessas tecnologias; a necessidade de um amplo escopo de recursos; e os problemas de coordenação entre tecnologias e distâncias geográficas. Esses achados vão ao encontro dos resultados que também mostram a diversificação tecnológica positivamente afetada pelo tamanho da firma e por seus estoques de conhecimento. Em outras palavras, as estratégias de diversificação para essas tecnologias são adotadas por firmas de grande porte e com elevada capacidade de acumulação de conhecimentos. Novas pesquisas nesse tema devem ser orientadas a novas variáveis que expliquem a exploração internacional dessas tecnologias e à ampliação da caraterização das firmas. 


\section{REFERÊNCIAS}

ALKEMADE, F. et al. Tracking the internationalization of multinational corporate inventive activity: National and sectoral characteristics. Research Policy, v. 44, n. 9, p. 1763-1772, 2015.

ARORA, A.; FOSFURI, A.; GAMBARDELLA, A. Markets for technology: The economics of innovation and corporate strategy. Cambridge: MIT Press, 2001.

BAUM, C. F. Stata tip 63: Modeling proportions. The Stata Journal, v. 8, n. 2, p. 299-303, 2008.

BERRY, C. H. Corporate growth and diversification. The Journal of Law \& Economics, v. 14, n. 2, p. 371-383, 1971.

BRESCHI, S.; LISSONI, F; MALERBA, F. Knowledge-relatedness in firm technological diversification. Research Policy, v. 32, n. 1, p. 69-87, 2003.

BUREAU VAN DIJK. Orbis Database. London: Bureau van Dijk, 2013. Disponível em: https:// orbis.bvdinfo.com. Acesso em: 2 jul. 2016.

CANTWELL, J. A.; FAI, F. Firms as the source of innovation and growth: The evolution of technological competence. Journal of Evolutionary Economics, v. 9, p. 331-366, 1999.

CANTWELL, J. A.; GAMBARDELLA, A.; GRANSTRAND, O. Technological and corporate diversification. In: CANTWELL, J. A.; GAMBARDELLA, A.; GRANSTRAND, O. (Eds.). The economics and management of technological diversification. London: Routledge, 2004. p. 1-17.

CASTELLACCI, F. Technological regimes and sectoral differences in productivity growth. Industrial and Corporate Change, v. 16, n. 6, p. 1105-1145, 2007.

CHANG, K.-C. et al. Nonlinear effect of technological diversification on the corporate patent performance. Journal of Applied Sciences, v. 14, n. 3, p. 273-278, 2014.

CHEN, J. H.; JANG, S. L.; WEN, S. H. Measuring technological diversification: Identifying the effects of patent scale and patent scope. Scientometrics, v. 84, n. 1, p. 265-275, 2010.

CHEN, Y. S.; CHANG, K. C. Using the entropy-based patent measure to explore the influences of related and unrelated technological diversification upon technological competences and firm performance. Scientometrics, v. 90, n. 3, p. 825-841, 2012.

CHEN, Y. S.; SHIH, C. Y.; CHANG, C. H. The effects of related and unrelated technological diversification on innovation performance and corporate growth in the Taiwan's semiconductor industry. Scientometrics, v. 92, n. 1, p. 117-134, 2012.

CHEN, Y.-M.; YANG, D.-H.; LIN, F.-J. Does technological diversification matter to firm performance? The moderating role of organizational slack. Journal of Business Research, v. 66, n. 10, p. 1970-1975, 2013.

CHIU, Y. C. et al. Technological scope: Diversified or specialized. Scientometrics, v. 82, n. 1, p. $37-58,2010$.

COHEN, W. M.; LEVIN, R. C. Empirical studies of innovation and market structure. In: SCHMALENSEE, R.; WILLIG, R. D. (Eds.). Handbook of industrial organization. North Holland: Elsevier, 1989. p. 1060-1107. 
COHEN, W. M.; LEVINTHAL, D. Absorptive capacity: A new perspective on learning and innovation. Administrative Science Quarterly, v. 35, n. 1, p. 128-152, 1990.

CORRADINI, C.; DE PROPRIS, L. Beyond local search: Bridging platforms and inter-sectoral technological integration. Research Policy, v. 46, n. 1, p. 196-206, 2016.

CORRADINI, C.; DEMIREL, P.; BATTISTI, G. Technological diversification within UK's small serial innovators. Small Business Economics, v. 47, n. 1, p. 163-177, 2016.

CORROCHER, N.; MALERBA, F.; MONTOBBIO, F. Schumpeterian patterns of innovative activity in the ICT field. Research Policy, v. 36, n. 3, p. 418-432, 2007.

COSTANTINI, V.; MAZZANTI, M. On the green and innovative side of trade competitiveness? the impact of environmental policies and innovation on EU exports. Research Policy, v. 41, n. 1, p. 132-153, 2012.

DE RASSENFOSSE, G. et al. The worldwide count of priority patents: A new indicator of inventive activity. Research Policy, v. 42, n. 3, p. 720-737, 2013.

DE RASSENFOSSE, G.; PALANGKARAYA, A.; WEBSTER, E. Why do patents facilitate trade in technology? Testing the disclosure and appropriation effects. Research Policy, v. 45, n. 7, p. 1326-1336, 2016.

DIBIAGGIO, L.; NESTA, L. Patents statistics, knowledge specialisation and the organisation of competencies. Revue d'Économie Industrielle, v. 110, n. 1, p. 103-126, 2005.

DOSI, G.; TEECE, D. J.; CHYTRY, J. Understanding industrial and corporate change: An introduction. In: DOSI, G.; TEECE, D. J.; CHYTRY, J. (Eds.). Understanding industrial and corporate change. Oxford: Oxford University Press, 2004. p. 10-21.

DU, J.; LU, J.; GUO, Y. Relationship between technological diversification of social network and technological innovation performance: Empirical evidence from China. Science Technology \& Society, v. 20, n.1, p. 60-88, 2015.

EUROPEAN PATENT OFFICE. EPO Worldwide Patent Statistical Database (EPO PATSTAT). Viena, 2012. Disponível em: https://data.epo.org. Acesso em: 7 mai. 2016.

GARCIA-VEGA, M. Does technological diversification promote innovation? An empirical analysis for European firms. Research Policy, v. 35, n. 2, p. 230-246, 2006.

GEELS, F. W. Reconceptualising the co-evolution of firms-in-industries and their environments: Developing an inter-disciplinary triple embeddedness framework. Research Policy, v. 43, n. 2, p. 261-277, 2014.

GRANSTRAND, O.; PATEL, P.; PAVITT, K. Multi-technology corporations: Why they have "distributed" rather than "distinctive core" competencies. California Management Review, v. 39, n. 4, p. 8-25, 1997.

HALL, B. H. A note on the bias in the herfindahl based on count data. Revue d'Économie Industrielle, v. 110, p. 149-156, 2005.

HALL, B. H.; JAFFE, A. B.; TRAJTENBERG, M. Market value and patent citations. The RAND Journal of Economics, v. 36, n. 1, p. 16-38, 2005.

HENDERSON, R. M.; COCKBURN, I. Scale, scope and spillovers: the determinats of research productivity in drug discovery. The RAND Journal of Economics, v. 27, n. 1, p. 32-59, 1996. 
HUANG, Y. F.; CHEN, C. J. The impact of technological diversity and organizational slack on innovation. Technovation, v. 30, n. 78, p. 420-428, 2010.

JANSEN, J. J. P.; VAN DEN BOSCH, F. A. J.; VOLBERDA, H. W. Managing potential and realized absorptive capacity: How do organizational antecedents matter? Academy of Management Journal, v. 48, n. 6, p. 999-1015, 2005.

KLEVORICK, A. K. et al. Policy in technological opportunities. Research in Economics, v. 24, p. $185-205,1995$.

KRAMMER, S. M. S. The role of diversification profiles and dyadic characteristics in the formation of technological alliances: Differences between exploitation and exploration in a low-tech industry. Research Policy, v. 45, n. 2, p. 517-532, 2016.

LAI, H. C. et al. Technological diversification and organizational divisionalization: The moderating role of complementary assets. British Journal of Management, v. 21, n. 4, p. 983-995, 2010.

LETEN, B.; BELDERBOS, R.; VAN LOOY, B. Technological diversification, coherence, and performance of firms. Journal of Product Innovation Management, v. 24, n. 6, p. 567-579, 2007.

LIN, C.; CHANG, C. C. The effect of technological diversification on organizational performance: An empirical study of S\&P 500 manufacturing firms. Technological Forecasting and Social Change, v. 90, n. B, p. 575-586, 2015.

MALERBA, F.; ORSENIGO, L. Technological regimes and firm behaviour. Industrial and Corporate Change, v. 19, n. 1, p. 45-71, 1993.

MONTGOMERY, C. A. The measurement of firm diversification: Some new empirical evidence. Academy of Management Journal, v. 25, n. 2, p. 299-307, 1982.

NELSON, R. R.; WINTER, S. G. Evolutionary theorizing in economics. Journal of Economic Perspectives, v. 16, n. 2, p. 23-46, 2002.

NESTA, L.; SAVIOTTI, P. P. Coherence of the knowledge base and the firm's innovative performance: Evidence from the U.S. pharmaceutical industry. The Journal of Industrial Economics, v. 53, n. 1, p. 123-142, 2005.

NOOTEBOOM, B. et al. Optimal cognitive distance and absorptive capacity. Research Policy, v. 36, n. 7, p. 1016-1034, 2007.

OECD - ORGANISATION FOR ECONOMIC CO-OPERATION AND DEVELOPMENT. OECD patent statistics manual. Paris: OECD Publishing, 2009.

OECD - ORGANISATION FOR ECONOMIC CO-OPERATION AND DEVELOPMENT. OECD Science, technology and industry outlook 2014. Paris: OECD Publishing, 2014.

PAPKE, L. E.; WOOLDRIDGE, J. M. Econometric methods for fractional response variables with an application to 401 (K) plan participation rates. Journal of Applied Econometrics, v. 11, n. 6, p. 619-632, 1996.

PATEL, P. R.; PAVITT, K. The technological competencies of the world's largest firms: Complex and path-dependent, but not much variety. Research Policy, v. 26, n. 2, p. 141-156, 1997.

PATEL, P.; PAVITT, K. The wide (and increasing) spread of technological competencies in the world's largest firms: A challenge to conventional wisdom. In: CHANDLER, A. D.; 
HAGSTROM, P.; SÖLVELL, Ö. (Eds.). The dynamic firm: The role of technology, strategy, organization, and regions. Oxford: Oxford University Press, 1998. p. 192-213.

PAVITT, K. Technologies, products and organization in the innovating firm: What Adam Smith tells us and Joseph Schumpeter doesn't. Industrial and Corporate Change, v. 7, n. 3, p. 433-452, 1998.

PAVITT, K.; ROBSON, M.; TOWNSEND, J. Technological accumulation, diversification and organisation in UK companies, 1945-1983. Management Science, v. 35, n. 1, p. 81-99, 1989.

PENROSE, E. T. The theory of the growth of the firm. 4. ed. Oxford: Oxford University Press, 1959.

PICCI, L.; SAVORELLI, L. Internationalized R\&D activities and technological specialization: An analysis of patent data. SSRN Electronic Journal, 2012.

PISCITELLO, L. Corporate diversification, coherence and economic performance. Industrial and Corporate Change, v. 13, n. 5, p. 757-787, 2004.

PODOLNY, J. M.; STUART, T. E. A role-based ecology of technological change. American Journal of Sociology, v. 100, n. 5, p. 1224-1260, 1995.

POPP, D. Induced innovation and energy prices. American Economic Review, v. 92, n. 1, p. 160-180, 2012.

QUINTANA-GARCÍA, C.; BENAVIDES-VELASCO, C. A. Innovative competence, exploration and exploitation: The influence of technological diversification. Research Policy, v. 37, n. 3, p. 492-507, 2008.

RAHKO, J. Internationalization of corporate R\&D activities and innovation performance. Industrial and Corporate Change, v. 25, n. 6, p. 1019-1038, 2016.

SCOTT, J. T. Purposive diversification and economic performance. Cambridge: Cambridge University Press, 1993.

SEBREK, S. S. Managing organisations in Schumpeterian environments: Intra-industry diversification through strategic technology alliances and patents. Technology Analysis \& Strategic Management, v. 27, n. 2, p. 161-181, 2015.

TEECE, D. J. et al. Understanding corporate coherence: Theory and evidence. Journal of Economic Behavior and Organization, v. 23, n. 1, p. 1-30, 1994.

TOH, P. K.; KIM, T. Why put all your eggs in one basket? A competition-based view of how technological uncertainty affects a firm's technological specialization. Organization Science, v. 24 , n. 4 , p. 1214-1236, 2013.

TORRISI, S.; GRANSTRAND, O. Technological and business diversification: A survey of theories and empirical evidence. In: CANTWELL, J. A.; GAMBARDELLA, A.; GRANSTRAND, O. (Eds.). The economics and management of technological diversification. London: Routledge, 2004. p. 21-68.

ZAHRA, S. A.; GEORGE, G. Absorptive capacity: A review, and extension. Academy of Management Review, v. 27, n. 2, p. 185-203, 2002. 